Check for updates

Cite this: RSC Adv., 2018, 8, 27349

Received 9th June 2018

Accepted 25th July 2018

DOI: $10.1039 / \mathrm{c} 8 \mathrm{ra04936j}$

rsc.li/rsc-advances

\section{Controllable synthesis of AgNWs@PDA@AgNPs core-shell nanocobs based on a mussel-inspired polydopamine for highly sensitive SERS detection $\uparrow$}

\begin{abstract}
Zhiliang Zhang, (D)*ac Tiantian Si, ${ }^{\text {b }}$ Jun Liu, ${ }^{a}$ Kehui Han ${ }^{\mathrm{b}}$ and Guowei Zhou ${ }^{* b}$
In this work, a series of AgNWs@PDA@AgNPs core-shell nanocobs based on a mussel-inspired polydopamine (PDA) were controllably synthesized and achieve highly sensitive SERS detection. Owing to the existence of abundant catechol and amine functional groups, PDA molecules could assemble a functional layer on the surface of silver nanowires (AgNWs) and exhibit exceptional adhesion performance. More importantly, silver nanoparticles (AgNPs) with controlled coverage and size were achieved on the surface of the PDA layer by in situ reduction of silver ions into AgNPs with catechol functional groups, forming AgNWs@PDA@AgNPs core-shell nanocobs. By regulating synergistical effect between the AgNWs and AgNPs, the AgNWs@PDA@AgNPs core-shell nanocobs demonstrated a highly sensitive and stable SERS response to Rhodamine 6G (R6G) molecules, and a low limit of detection down to $10^{-12} \mathrm{M}$. Furthermore, the AgNWs@PDA@AgNPs core-shell nanocobs showed an excellent reproducibility and superior stability as a SERS substrate to achieve trace detection. This strategy would have great potential to fabricate multifarious SERS-active substrates that make it possible to detect single molecules and singles cell in chemical and biological fields.
\end{abstract}

\section{Introduction}

SERS is a powerful and efficient vibrational spectroscopic technique for detecting molecules adsorbed on or near the surface of certain nanostructures, and provides molecular structural information..$^{1-3}$ In recent years, as a label-free analytical technique, SERS has attracted great interest owing to its high sensitivity and excellent selectivity, and single molecule level detection has been achieved in the fields of chemistry and biology. ${ }^{4-6}$ In theory, the SERS effect is closely related to localized surface plasmon resonance (LSPR) and would greatly enhance the Raman signals when Raman probe molecules are located at the interstice or junction of aggregates with multiple nanostructures. The enormous electromagnetic (EM) enhancement is mainly derived from the plasmon coupling among the junction sites or interstice of the nanostructures in close proximity, and would be called SERS "hot spots".,

At present, the most commonly used SERS substrates are noble metal nanomaterials, such as gold, silver and copper

\footnotetext{
${ }^{a}$ State Key Laboratory of Biobased Material and Green Papermaking, Qilu University of Technology, Shandong Academy of Sciences, Jinan, 250353, P. R. China

${ }^{b}$ School of Chemistry and Pharmaceutical Engineering, Qilu University of Technology, Shandong Academy of Sciences, Jinan 250353, China

${ }^{c}$ Beijing National Laboratory for Molecular Sciences (BNLMS), Institute of Chemistry, Chinese Academy of Science, Beijing 100190, China. E-mail: zhzhl@iccas.ac.cn

$\dagger$ Electronic supplementary information (ESI) available. See DOI: 10.1039/c8ra04936j
}

nanostructures, which could generate a strong local electric field with the excitation of incident light. ${ }^{9}$ Compared with the other metallic nanomaterials, silver nanostructures are more widely used as the SERS activity substrate that benefits from their wide range of plasmon resonance effects in the visible region, great signal enhancement and facile synthesis process. $^{5,7,10}$ So far, silver nanostructures with various morphologies, such as nanospheres, ${ }^{11}$ nanorods, ${ }^{12}$ nanowires ${ }^{\mathbf{1 3}}$ and nanosheets, ${ }^{\mathbf{1 4}}$ have been reported as SERS-active substrates and achieve highly sensitive molecules detection. AgNWs have a large surface area as a one-dimensional nanostructure and high crystallinity enabling them to be an ideal SERS substrate for large-scale sensing application. ${ }^{\mathbf{1 5 , 1 6}}$ But there existed a problem that momentum is seriously mismatched between the propagating plasmon and photon on the smooth longitudinal surface. ${ }^{15,16}$ As a result, the optical coupling into the plasmon modes is very weak at visible-light frequency and the SERS "hot spots" are strictly confined to the sharp AgNWs tips. ${ }^{17,18}$

To resolve this challenge, a variety of approaches have been proposed to increase nanogaps and sharp edges as active SERS "hot spots" on AgNWs longitudinal length and achieve highly sensitive SERS detection. ${ }^{\mathbf{8 , 1 5 , 1 8 - 2 4}}$ In general, as the incident lights are polarized transverse to the corrugated AgNWs or AgNWs decorated with nanoparticles, a strongest polarizationdependent scattering would occur on these one-dimensional AgNWs surface. Theoretical calculations have demonstrated that the spacing of adjacent nanoparticles on the surface of 
AgNWs can provide excellent Raman enhancement, and the largest SERS enhancement is likely located at nanogaps and interstitial junctions between these metallic nanostructures. ${ }^{25-27}$ In this context, the methods such as decorating AgNWs with small metallic nanoparticles, ${ }^{18,21-23,28}$ chemical etching to roughen the AgNWs surface ${ }^{15}$ or assembling AgNWs into 2D multilayered arrays, have been demonstrated that SERS active "hot spots" increase significantly along the longitudinal axis, ${ }^{17,20,24}$ therefore dramatically increasing the strong SERS effect.

For instance, Tian group fabricated a series of corrugated polycrystalline AgNWs through a three-dimensional (3D) electrochemical deposition, and the enhancement factor of corrugated nanowires was 1-2 orders of magnitude higher than these smooth AgNWs. Moreover, adhesion nanoparticles on the surface of the corrugated AgNWs could further enhance the enhancement factor as a result of the coupling between the continua plasmon of the corrugated AgNWs and discontinuous plasmon of the AgNPs. ${ }^{21}$ Lu et al. developed a plasmonmediated photochemical method to etch and roughen the AgNWs surface morphology and made the intensity of Raman scattering increase dramatically. These results indicated that the SERS enhancement was not only related to the structure of as-fabricated nanomaterials, but also related to surface morphological changes that caused by plasmon-mediated photochemical reactions. $^{8}$ Chen et al. utilized a LangmuirBlodgett technique to assemble AgNPs into 3D woodpile-like platforms, along with the assembled AgNWs layer from monolayer to three layers, and the SERS enhancement factor raised from $3.1 \times 10^{3}$ to $2.6 \times 10^{4}$ respectively. ${ }^{17}$ The SERS-active substrates by these fabrication methods displayed ultra high sensitivity and perfect reliability, however, the fabrication processes tended to be relatively complicated, tedious and timeconsuming. So far, it still remains a great challenge to fabricate an AgNWs-based SERS-active substrate with controllable Raman "hot spots" which can obviously improve the detection sensitivity and reliability in an efficient and versatile approach.

Dopamine (DA) have abundant catechol and amine active groups in the molecules surface and could serve as a versatile platform owing to their distinctive properties, for instance, selfpolymerization, chemical recognition and anchor capability, which is very similar to the mussels in molecules structure and property. ${ }^{29}$ Especially, the self-polymerization of DA could provide a facile and efficient way to surface modification and generate an extraordinarily robust adhesive layer via immersing the substrates into the solution. More importantly, owing to the existence of abundant catechol and amine groups on the surface, PDA molecules could have fairly strong complexing actions with metallic ions and spontaneously in situ reduce them into the metallic nanoparticles by oxidizing catechol into the corresponding quinine groups under alkaline aqueous solution. ${ }^{30-32}$ As a result, a uniform metallic nanostructures with a desirable size and coverage are facilely deposited on all types of substrates, even including superhydrophobic surface, which provide a promising opportunity to construct nanogaps and sharp edges as controllable Raman "hot spots" on the AgNWs surface.
Therefore, inspired by these unique properties of PDA, in this contribution, an efficient and versatile strategy was developed to controllably synthesize a series of AgNWs@PDA@AgNPs core-shell nanocobs based on mussel-inspired PDA molecules and achieved highly sensitive SERS detection. By regulating the catechol groups and $\left[\mathrm{Ag}\left(\mathrm{NH}_{3}\right)_{2}\right]^{+}$ions, AgNPs with controlled coverage and size were decorated on surface of PDA layer by the in situ reduction silver ions into AgNPs with catechol functional groups. These AgNWs@PDA@AgNPs core-shell nanocobs could provide a great number of SERS "hot spots" and display a superior controllability, ultra-high sensitivity and excellent reproducibility by regulating synergistical effect between the AgNPs and AgNWs along the longitudinal axis. It was identified that the limit of detection was down to $10^{-12} \mathrm{M}$ using R6G as probe molecule. It would demonstrate a great potential to fabricate multifarious SERS-active substrates by this fabrication strategy and achieve a reliable and sensitive detection in chemical and biological fields.

\section{Experimental}

\section{Chemicals and materials}

Dopamine hydrochloride, tris-base ( $\geq 99.9 \%$ ), ethylene glycol (EG), copper chloride $\left(\mathrm{CuCl}_{2}\right)$, cuprous chloride $(\mathrm{CuCl})$, silver nitrate $\left(\mathrm{AgNO}_{3}\right)$ and polyvinylpyrrolidone $\left(\mathrm{PVP}, M_{\mathrm{w}}=4 \times 10^{4}\right)$ were purchased from Sigma-Aldrich. Hydrochloric acid, ethanol, acetone and R6G were purchased from Beijing Chemical Co. The rest reagents and materials were analytical grade and without further purification, and the ultrapure (18.2 $\mathrm{M} \Omega$ ) water used throughout the entire experiment was produced by the Milli-Q system.

\section{Controllable synthesis of AgNWs in polyol process}

The AgNWs with controlled diameter and length were synthesized in polyol process according to the previous published literature. ${ }^{33}$ In the process of reaction, EG $(50 \mathrm{~mL})$ was added to a three-necked flask, and heated to $150{ }^{\circ} \mathrm{C}$ under magnetic stirring at $200 \mathrm{rpm}$, and the prepared Cu-additive $\left(\mathrm{CuCl}_{2}\right.$ and $\mathrm{CuCl}$ ) solution was added to the heated EG solution. The PVP solution dissolved in EG $(15 \mathrm{~mL}, 0.15 \mathrm{M}$, the concentration calculated on the basis of the repeating unit) was added into the three-necked flask drop by drop, following by added $\mathrm{AgNO}_{3}$ solution dissolved in EG $(15 \mathrm{~mL}, 0.1 \mathrm{M})$. The reaction system was reacted for 1-1.5 $\mathrm{h}$ at the temperature and gradually became opaque gray. By centrifugation at $6000 \mathrm{rpm}$, AgNWs were collected from the solution and stored in ethanol for further use in the experiments.

\section{Controllable synthesis of AgNWs@PDA nanostructures}

To a mixture of the AgNWs and Tris-HCl buffer solution (10 $\mathrm{mM}, \mathrm{pH}=8.5$ ), the dopamine hydrochloride aqueous solution was added into above mixture carefully with a mildly stirring speed and reacted for about 24 hours at room temperature. After the reaction, the solution color was changed from gray to brown, and the redundant PDA was removed by dumping the upper suspension from the AgNWs precipitate. 
After centrifuging at $6000 \mathrm{rpm}$ and washing with ultrapure water three times, the AgNWs@PDA were obtained and stored in ethanol for further use.

\section{Controllable Synthesis of AgNWs@PDA@AgNPs nanocobs}

After immersing the AgNWs@PDA into a fresh $\left[\mathrm{Ag}\left(\mathrm{NH}_{3}\right)_{2}\right]^{+}$ aqueous solution, the mixed reactants were reacted to decorate AgNPs on AgNWs surface at room temperature with a moderate stirring speed. According to reaction ratio, the concentration of $\left[\mathrm{Ag}\left(\mathrm{NH}_{3}\right)_{2}\right]^{+}$aqueous solution was controlled in the range of 1.2 $\times 10^{-2} \mathrm{M}$ to $4.8 \times 10^{-2} \mathrm{M}$. After the reaction was complete, the AgNWs@PDA@AgNPs were obtained by centrifugation and stored in ethanol for SERS detection.

\section{Characterization}

The Bruker D8 X-ray diffractometer and Hitachi U-4100 spectrophotometer were used to collect the UV-vis absorption and Xray diffraction (XRD) spectra of AgNWs, AgNWs@PDA and AgNWs@PDA@AgNPs respectively. The Hitachi S-4800 scanning electron microscope was used to characterize the microscopic morphology and energy dispersive X-ray spectroscopy (EDS) of above samples, and JEOL JEM-2100F transmission electron microscopy (TEM) was used to observe the morphology and particle size of the samples. Thermo Scientific ESCALab 250Xi using $200 \mathrm{~W}$ monochromatic Al $\mathrm{K} \alpha$ radiation was to record X-ray photoelectron spectroscopy (XPS) data. Raman spectra of the prepared samples were measured by Renishaw inVia9 Raman microscope, and use a $50 \times$ objective and $532 \mathrm{~nm}$ laser irradiation to focused laser beam into a spot with a diameter of about $1 \mu \mathrm{m}$. The intensity of irradiation laser was controlled at $1 \mathrm{~mW}$ to activate the samples during the whole process, and all SERS signals were gathered about 10 s. SERS characterization was through dripping R6G aqueous solution onto the surface of the prepared SERS active substrate and dried under ambient condition.

\section{Results and discussion}

In order to obtain highly sensitive and efficient SERS detection, it was very crucial to fabricate the SERS substrate with sufficient Raman hot spots. As illustrated in Fig. 1, a controllable-density AgNPs on AgNWs surface based on PDA molecules as Raman hot spots was designed and fabricated for target molecules detection. Briefly, AgNWs with a controllable diameter and length were synthesized in polyol process reaction, and exhibited a smooth surface. Due to the exceptional interface adhesion, DA could be easily deposited on the AgNWs surface by a spontaneous self-polymerization in a weakly alkaline aqueous solution and formed a functional PDA layer with a rich catechol and amine groups. As the catechol and amine groups in PDA molecules could strongly complexed with $\left[\mathrm{Ag}\left(\mathrm{NH}_{3}\right)_{2}\right]^{+-}$ ions, a controllable AgNPs decoration on AgNWs surface gradually emerged utilized oxidizing catechol into the corresponding quinine groups with the in situ reduction of $\left[\mathrm{Ag}\left(\mathrm{NH}_{3}\right)_{2}\right]^{+}$ions into AgNPs. As a results, a series of AgNWs@PDA@AgNPs core-shell nanocobs with controllable AgNPs size and distribution were successfully synthesized by regulating the concentration of $\left[\mathrm{Ag}\left(\mathrm{NH}_{3}\right)_{2}\right]^{+}$ions and PDA molecules. These AgNWs@PDA@AgNPs core-shell nanocobs would serve as SERS-active substrates after dipping the target molecules, and displayed high sensitivity and reliable stability.

The surface plasmon resonance of nanostructures was highly sensitive to the morphology, component and dielectric environment around them. Therefore, UV-visible spectra were performed to characterize AgNWs, AgNWs@PDA and AgNWs@PDA@AgNPs respectively. From Fig. 2a, two distinct surface plasmon resonance peaks appeared at about 350 and $380 \mathrm{~nm}$ in the absorption spectrum of AgNWs. The peak at $350 \mathrm{~nm}$ was assigned to longitudinal plasmon resonance absorption of the AgNWs, while the peak at $380 \mathrm{~nm}$ was the transverse plasmon resonance of AgNWs. ${ }^{34}$ Both of these peaks almost disappeared as AgNWs were deposited a PDA layer by

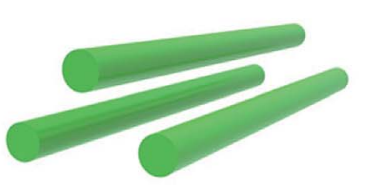

AgNWs

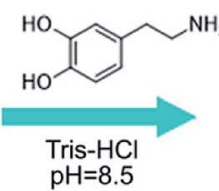

$\mathrm{pH}=8.5$

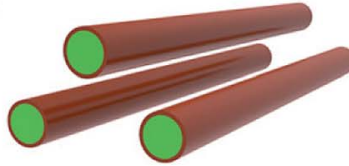

AgNWs@PDA

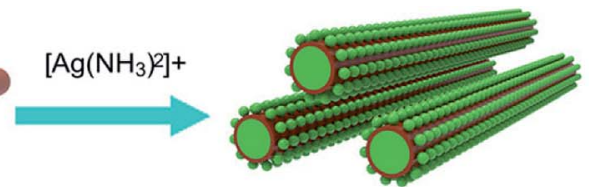

AgNWs@PDA@AgNPs

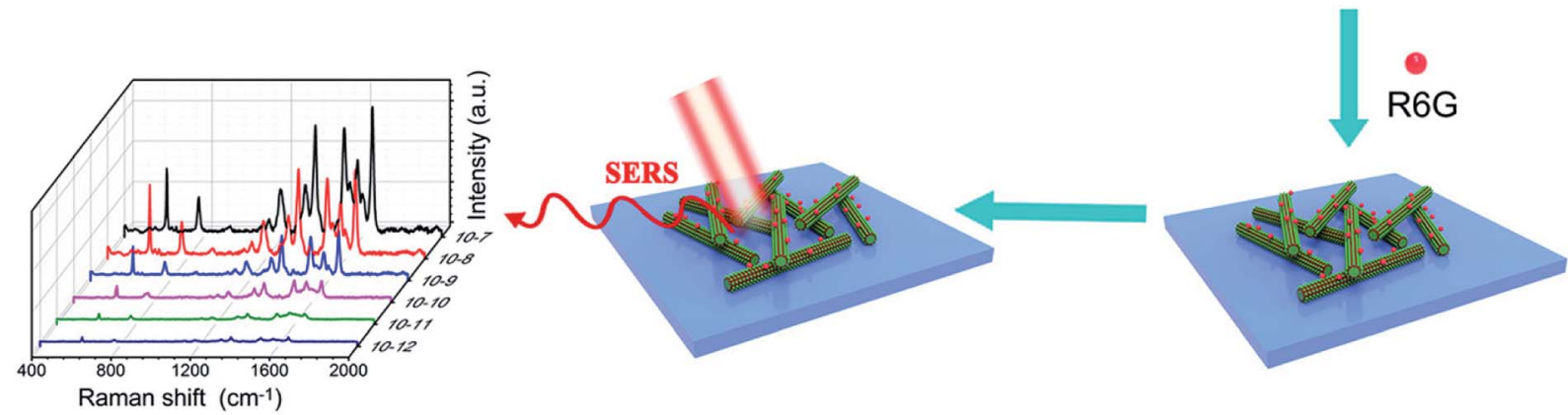

Fig. 1 Schematic illustration of the construction of AgNWs@PDA@AgNPs nanocobs for highly sensitive SERS detection. 

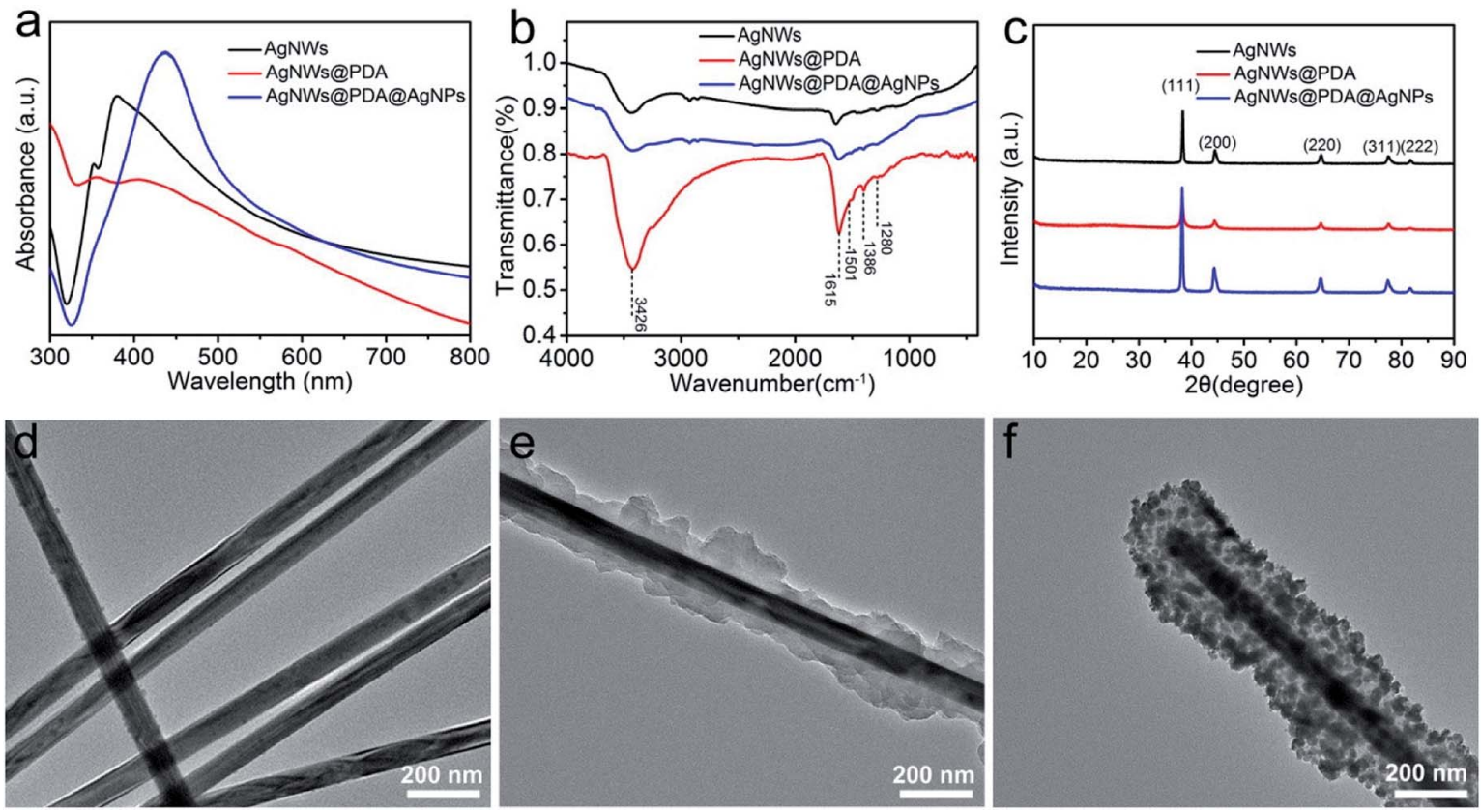

Fig. 2 (a) UV-vis, (b) FTIR and (c) XRD spectra of AgNWs, AgNWs@PDA and AgNWs@PDA@AgNPs, TEM images of (d) AgNWs, (e) AgNWs@PDA and (f) AgNWs@PDA@AgNPs respectively.

self-polymerization. After mixing with silver ammonia solution, a typical broad absorption peak at $440 \mathrm{~nm}$ was clearly observed, indicating the formation of abundant AgNPs decorated on the AgNWs surface.

FTIR spectra were also employed to investigate the formation process of AgNWs@PDA@AgNPs. In contrast with the spectra of AgNWs and AgNWs@PDA@AgNPs in Fig. 2b, the spectrum of AgNWs@PDA exhibited a characteristic resonance peaks at $3426 \mathrm{~cm}^{-1}$ and could be attributed to the stretching vibrations of $-\mathrm{NH}_{2}$ and $-\mathrm{OH}$ groups. The typical peak at $1280 \mathrm{~cm}^{-1}$ was assigned to $\mathrm{C}-\mathrm{O}$ groups stretching vibration in PDA molecules. Similarly, the absorption peaks at $1615 \mathrm{~cm}^{-1}, 1501 \mathrm{~cm}^{-1}$ and $1386 \mathrm{~cm}^{-1}$ were attributable to the stretching vibrations of $\mathrm{C}=$ $\mathrm{N}, \mathrm{C}-\mathrm{N}-\mathrm{C}$, and $\mathrm{C}=\mathrm{C}$ functional groups, originating from indoline or indole groups present in AgNWs@PDA. Moreover, the five characteristic peaks $\left(2 \theta=38.1^{\circ}, 44.2^{\circ}, 64.4^{\circ}, 77.4^{\circ}\right.$ and 81.5 ${ }^{\circ}$, Fig. 2c) in the AgNWs, AgNWs@PDA and AgNWs@PDA@AgNPs XRD spectra were attributed to (111), (200), (220), (311) and (222) crystalline plans of face centered cubic crystal structure, which matched well with the database of silver (JCPDS no. 83-0718). From the XRD spectrum in Fig. S1a, $\uparrow$ it could be also noticed that the AgNWs@PDA samples displayed a broad peak in the range of $20-30^{\circ}$, ascribed to the diffraction of the amorphous PDA structures on the AgNWs surface.

In order to obtain more detailed structural information, TEM was used to further investigate the AgNWs, AgNWs@PDA and AgNWs@PDA@AgNPs. From Fig. 2d-f, it was clearly seen that PDA could be anchored onto AgNWs surface and formed core-shell nanostructures, in which AgNWs served as a core and PDA formed an amorphous shell (Fig. 2e). As the $\left[\mathrm{Ag}\left(\mathrm{NH}_{3}\right)_{2}\right]^{+}$ ions were added to the AgNWs@PDA solution, it was interesting to note that a controllably-density AgNPs were emerged on the
AgNWs surface and formed a series of AgNWs@PDA@AgNPs nanocobs. From Fig. S1b-d, $\uparrow$ the aspect ratio of pristine AgNWs did not modify after PDA complexation and AgNPs formation. All these results proved that DA molecules could deposit on the AgNWs surface and assembled a functional PDA layer by selfpolymerization, and subsequently, the AgNPs could be formed on the AgNWs surface by in situ oxidizing catechol into the corresponding quinine groups.

In order to further elucidate the surface elemental composition, XPS was employed to evaluate the AgNWs, AgNWs@PDA and AgNWs@PDA@AgNPs samples. From Fig. 3a, only Ag, C, N and $\mathrm{O}$ elements spectra were obviously detected in the XPS survey scan spectra of AgNWs, AgNWs@PDA and AgNWs@PDA@AgNPs, and demonstrated the high purity characteristics of our samples. The disappearance of Ag3 $\mathrm{p}_{3 / 2}, \mathrm{Ag} 3 \mathrm{p}_{1 / 2}$ and $\mathrm{Ag} 3 \mathrm{~s}$ peaks suggested that PDA was successfully deposited on the AgNWs surface by self-polymerization. High resolution XPS spectra were also analyzed to verify the electronic status of each element exactly. The C1s spectrum (Fig. $3 \mathrm{~b}$ ) could be curvefitted with four peaks located at 284.5, 285.2, 286.2 and $288.2 \mathrm{eV}$, indicating the existence of four electronic states of carbon, which were originated from $\mathrm{C}-\mathrm{C}, \mathrm{C}-\mathrm{N}, \mathrm{C}-\mathrm{O}$ and $\mathrm{C}=\mathrm{O}$ in the PDA molecules. From Fig. 3c, two different peaks centered at $399.4 \mathrm{eV}$ and $399.9 \mathrm{eV}$ were observed in the curve-fitted N1s spectra of AgNWs@PDA@AgNPs, which was corresponded to $-\mathrm{N}=$ and $-\mathrm{N}-\mathrm{H}-$ in the PDA molecules. ${ }^{31}$ From Fig. $3 \mathrm{~d}$, it could also be seen that two distinct peaks the Ag3d spectrum were located at 368 and $374 \mathrm{eV}$, which represented the binding energies of spin-orbit for $\mathrm{Ag} 3 \mathrm{~d}_{5 / 2}$ and $\mathrm{Ag} 3 \mathrm{~d}_{3 / 2}$ respectively. The narrow width of the Ag3d peak indicated that only existence of the silver on the surface in the samples, which also provided a direct evidence for successful reduction of AgNPs. 

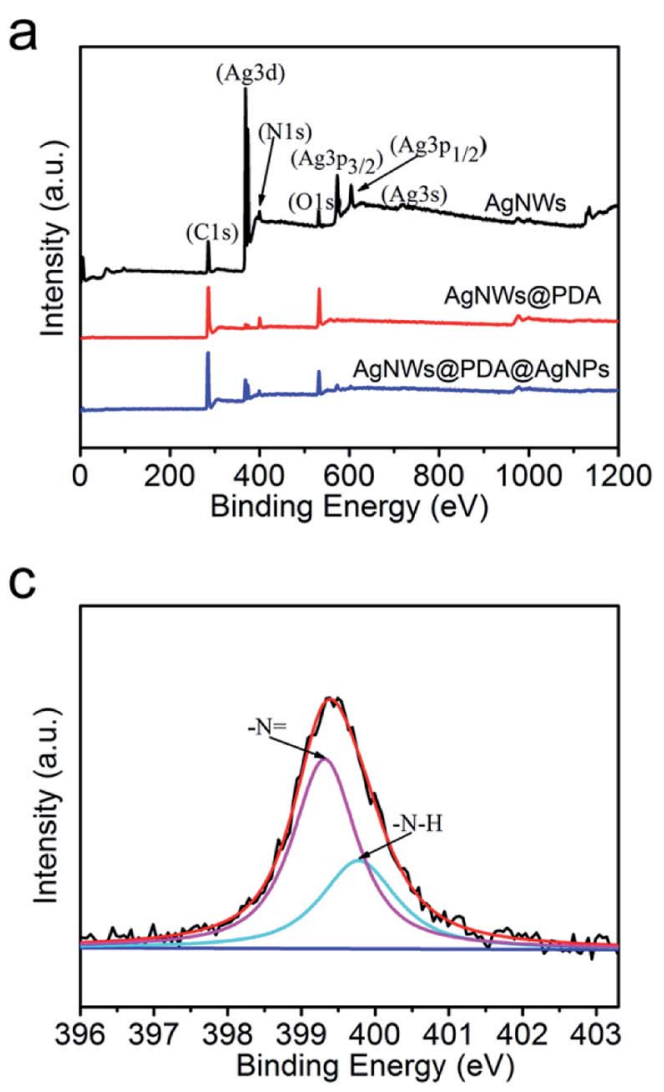
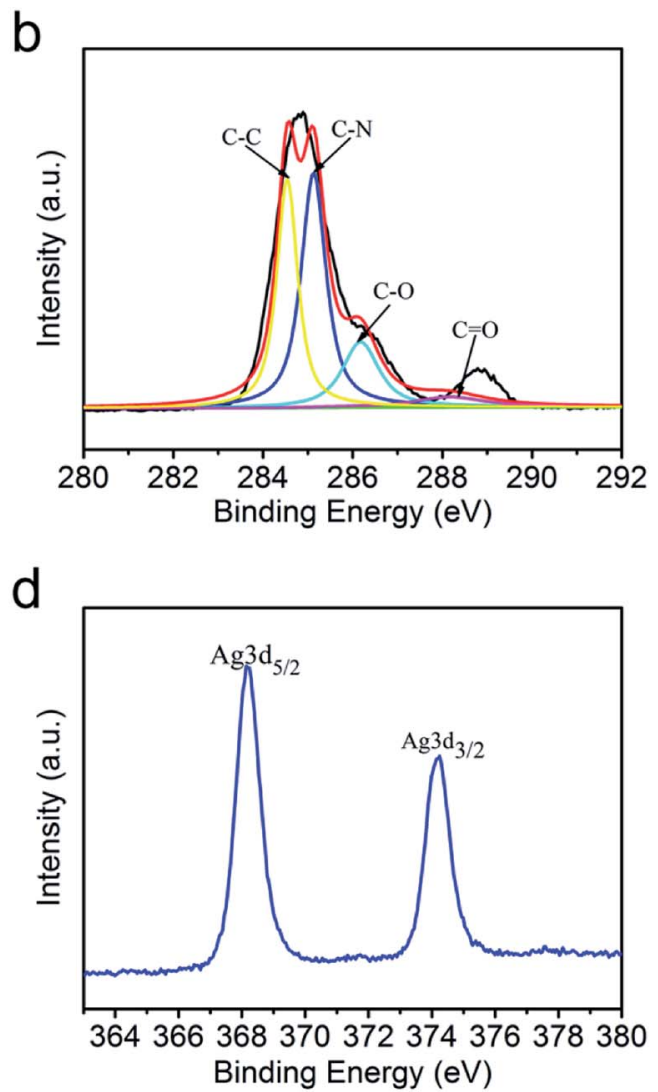

Fig. 3 (a) XPS survey of the AgNWs, AgNWs@PDA and AgNWs@PDA@AgNPs, (b) the C1s spectrum, (c) N1s spectrum and (d) Ag3d spectrum of AgNWsaPDA@AgNPs.

Moreover, the elements variation of synthesized sample at different stages by EDS was shown in Fig. 4a-f. Compared with Fig. 4d, the peaks of $\mathrm{C}, \mathrm{O}$ and $\mathrm{N}$ elements exhibited a significant enhancements in the spectrum of the AgNWs@PDA (Fig. 4e), and suggested that PDA layer was successful assembled on AgNWs surface (Fig. 4b). By contrast, the intensity of the C, N and $\mathrm{O}$ peaks declined sharply and the $\mathrm{Ag}$ peak increased oppositely after the AgNWs@PDA was dipped into the $\left[\mathrm{Ag}\left(\mathrm{NH}_{3}\right)_{2}\right]^{+}$solution (Fig. $4 \mathrm{f}$ ), which were attributed to the abundant AgNPs formation on the AgNWs@PDA surface (Fig. 4c). These EDS results were well consistent with the above TEM, XPS and SEM results. All these analysis demonstrated that the AgNWs@PDA@AgNPs nanocobs could be fabricated with this simple approach, which was favorable to construct a highly sensitive AgNWs SERS-active substrate with controllable Raman hot spots.

The morphology and distribution of AgNPs on the AgNWs surface were extremely pivotal to achieve a highly sensitive SERS detection, and AgNWs, AgNWs@PDA and AgNWs@PDA@AgNPs were examined by SEM respectively. From Fig. 5a, the AgNWs demonstrated a uniform straight morphology and smooth surface. In contrast to AgNWs with a smooth surface, the AgNWs@PDA exhibited a relatively rough and amorphous morphology (Fig. 5b), which indicated that PDA molecules deposited on the AgNWs surface and formed a functional layer. From Fig. 5c, after dipping AgNWs@PDA into $\left[\mathrm{Ag}\left(\mathrm{NH}_{3}\right)_{2}\right]^{+}$ solution for a certain time, almost all of AgNWs@PDA have successfully been decorated with AgNPs and formed a series of AgNWs@PDA@AgNPs core-shell nanocobs. It can be seen from Fig. 5c that the scattered AgNPs adhered tightly on the PDA surface and formed a corrugated surface. PDA served as a bridge molecules to connect AgNWs and AgNPs, and regulated the distribution and particle size of AgNPs on the surface of AgNWs@PDA@AgNPs.

These Ag-based nanostructures could be used to fabricate reliable SERS substrates to detect single molecule, and the Raman enhancement effect was verified by using R6G at a concentration of $1 \times 10^{-6} \mathrm{M}$. Fig. $5 \mathrm{~d}$ displayed the representative SERS spectra of R6G molecules on these three samples, and the peaks located at $612,774,1185,1311,1360,1511,1577$ and $1650 \mathrm{~cm}^{-1}$ were clearly observed, which matched well with the previous reports of R6G. ${ }^{35,36}$ It was noticed that the AgNWs@PDA@AgNPs nanocobs exhibited more remarkable Raman enhancement compared to AgNWs and AgNWs@PDA. For the AgNWs@PDA, the PDA layer might weak the SERS property in a certain extent. The signal of a small amount of probe molecules could still be detected at the areas near the inner AgNWs through such a PDA layer, which was proved by the SERS signal of AgNPs@PDA in Fig. S2. $\dagger$ For comparison, the R6G detection limit on the AgNWs@PDA SERS substrate was 1 $\times 10^{-8} \mathrm{M}$, and higher than $1 \times 10^{-10} \mathrm{M}$ on AgNWs SERS substrate. In fact, it could generate a huge local electromagnetic 

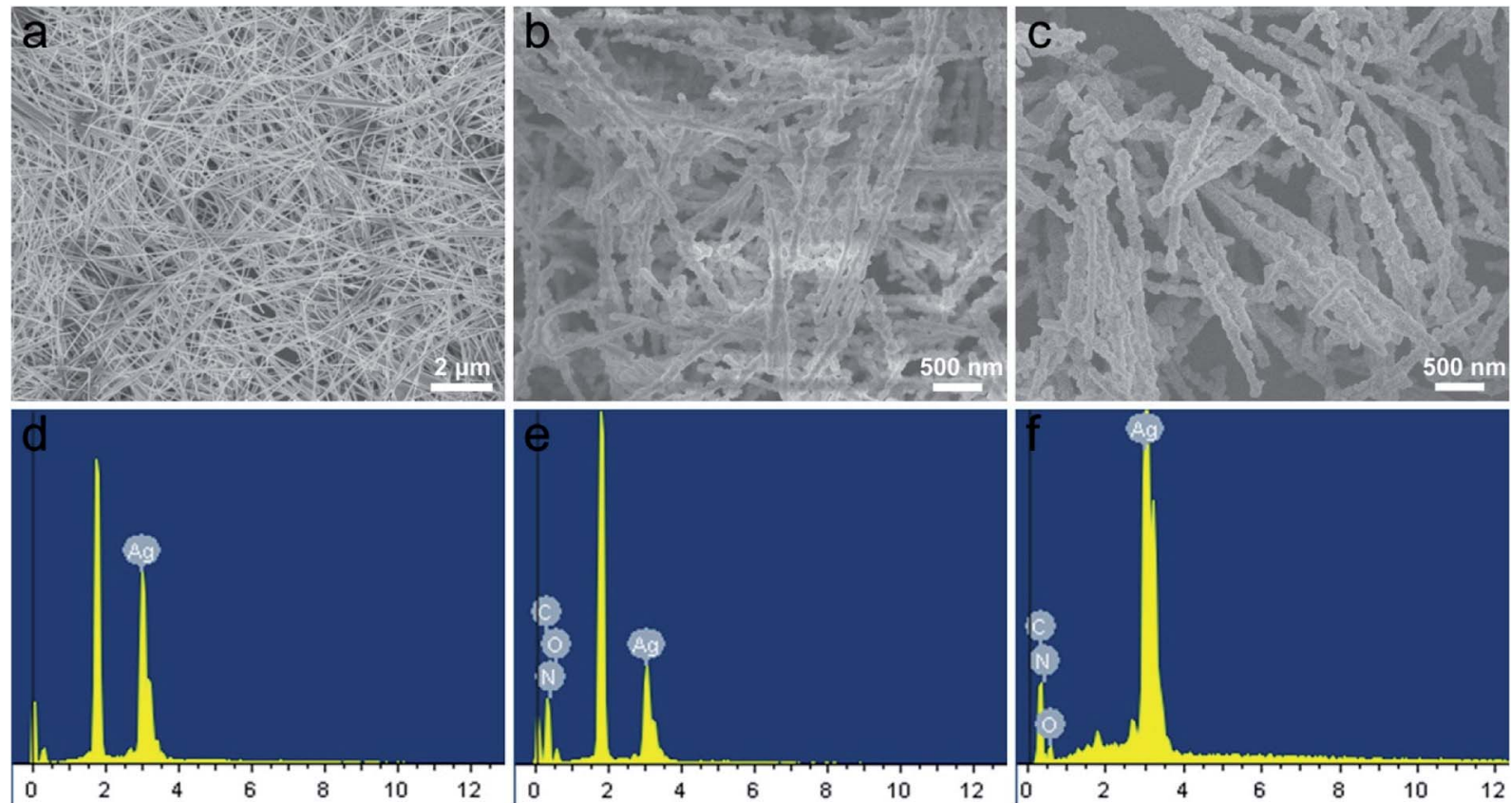

Fig. 4 SEM images of (a) AgNWs, (b) AgNWs@PDA and (c) AgNWs@PDA@AgNPs, the responding EDS of (d) AgNWs, (e) AgNWs@PDA and (f) AgNWs@PDA@AgNPs respectively.
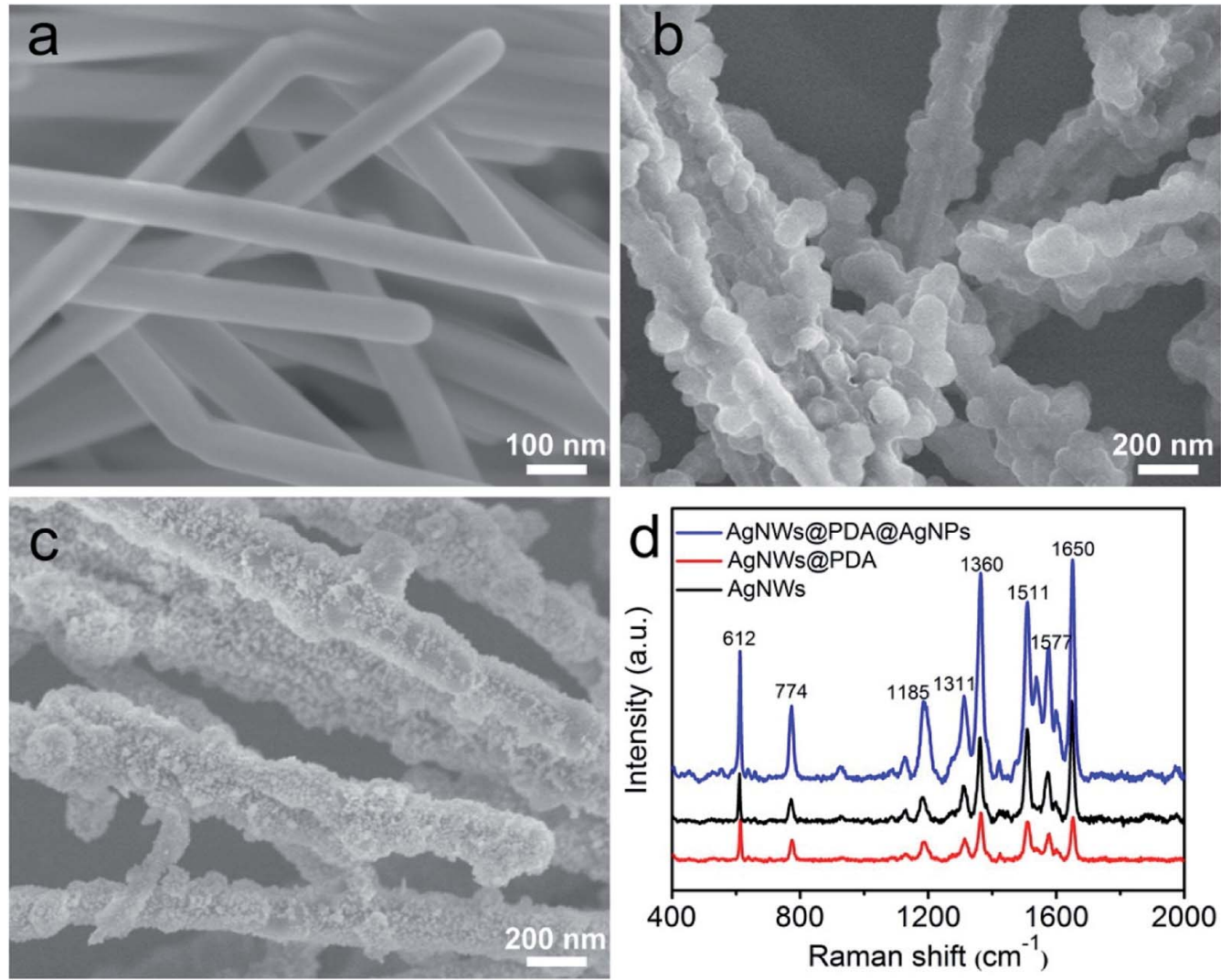

Fig. 5 SEM images of (a) AgNWs, (b) AgNWs@PDA and (c) AgNWs@PDA@AgNPs, (d) Raman spectra of R6G molecules (1 × $10^{-6}$ M) on AgNWs, AgNWs@PDA and AgNWs@PDA@AgNPs. 

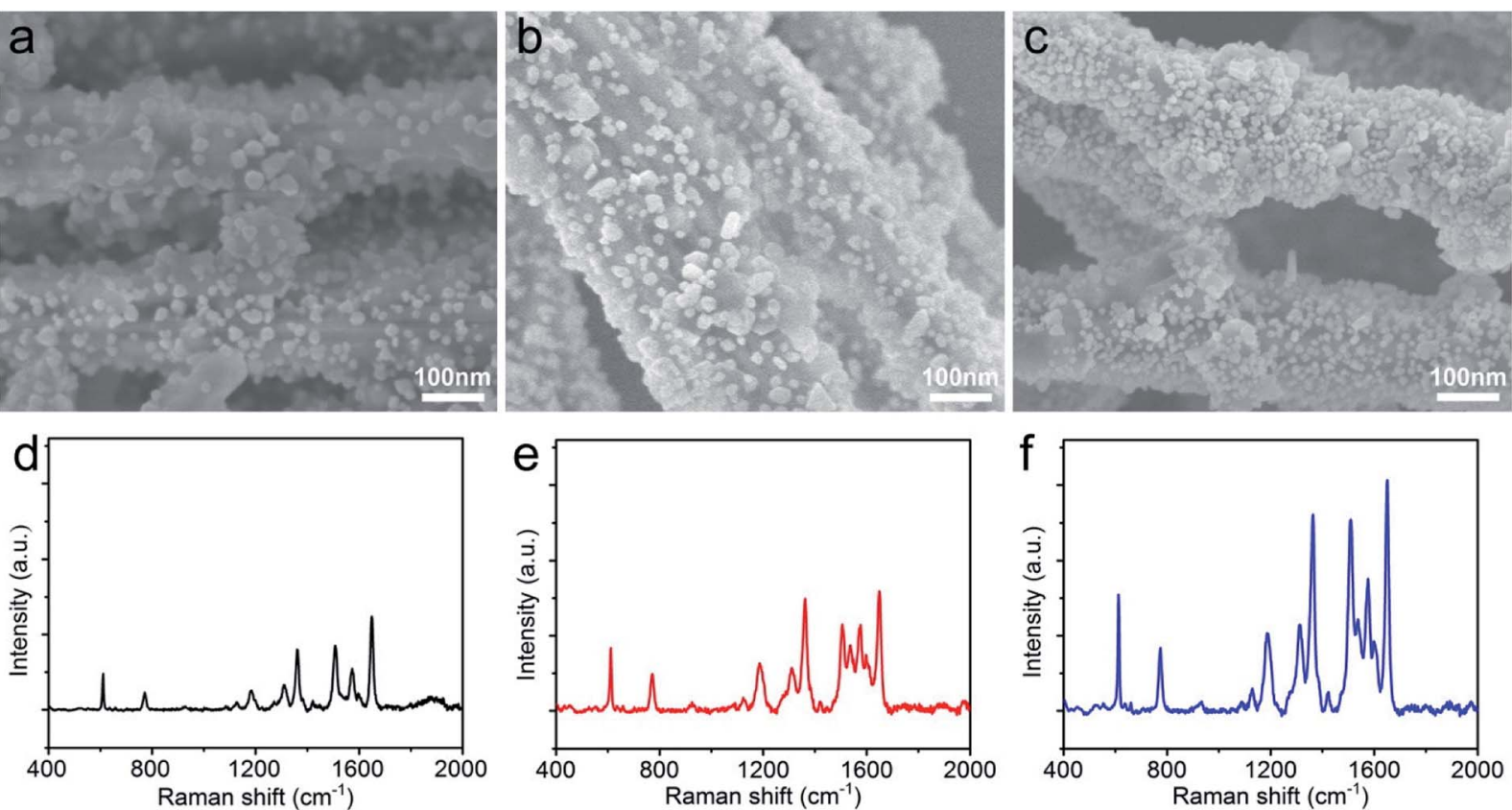

Fig. 6 SEM images of AgNWs@PDA@AgNPs synthesized at (a) $1.2 \times 10^{-2} \mathrm{M}$, (b) $2.4 \times 10^{-2} \mathrm{M}$, and (c) $4.8 \times 10^{-2} \mathrm{M}$ of $\left[\mathrm{Ag}\left(\mathrm{NH}_{3}\right)_{2}\right]^{+}$ions, respectively. $(\mathrm{d}-\mathrm{f})$ Raman spectra of $\mathrm{R} 6 \mathrm{G}\left(1 \times 10^{-6} \mathrm{M}\right)$ dripped on the corresponding AgNWs@PDA@AgNPs substrates.

field because of the interparticles electromagnetic coupling of the aggregated AgNPs, and offered a great Raman signal enhancements to achieve single-molecule level detection..$^{37,38}$

From the SERS spectra of R6G molecules on AgNWs, AgNWs@PDA, AgNWs@PDA@AgNPs and AgNPs in Fig. S3a, $\dagger$ the AgNPs substrate could produce excellent enhancement effect, and the R6G detection limit on the AgNPs SERS substrate was about $1 \times 10^{-11} \mathrm{M}$ (Fig. S3b $\dagger$ ). In the AgNWs@PDA@AgNPs nanocobs, abundant SERS "hot spots" were originated from the nanogaps and interstices of aggregate AgNPs and AgNWs, which were synergistically contributed to the high SERS activity. Moreover, such small nanogaps and interstices among adjacent AgNPs in AgNWs@PDA@AgNPs nanocobs were fairly beneficial to attain a highly strong SERS signal. As a result, the AgNWs@PDA@AgNPs nanocobs exhibited a superior SERS activity compared to the AgNWs and AgNWs@PDA nanostructures at the same R6G concentration.

The enhancement intensity of SERS was closely related to the LSPR, and it could be controlled by the properties of plasmonic nanostructures such as the size, shape and distribution. In order to regulate the size and distribution of AgNPs on the AgNWs@PDA surface, different concentrations of silver ammonia solution were used to synthesized a series of AgNWs@PDA@AgNPs nanocobs. From SEM images in Fig. 6a$\mathrm{c}$, as the concentration of silver ammonia solution was $1.2 \times$ $10^{-2} \mathrm{M}$, only a few part of AgNPs were immobilized on AgNWs@PDA surface and formed a low surface coverage (Fig. 6a). Along with the increasing concentration of $\left[\mathrm{Ag}\left(\mathrm{NH}_{3}\right)_{2}\right]^{+}$ ions, the surface coverage with AgNPs was gradually increased due to more $\left[\mathrm{Ag}\left(\mathrm{NH}_{3}\right)_{2}\right]^{+}$ions into AgNPs through the in situ reduction with catechol functional groups (Fig. 6b). As the concentration of silver ammonia solution further increased to $4.8 \times 10^{-2} \mathrm{M}$, a controllable-density AgNPs were formed and abundant nanogaps and interstices between adjacent AgNPs were emerged (Fig. 6c). From Fig. 6d-f, the Raman enhancement effect increased obviously followed by the increased concentration of $\left[\mathrm{Ag}\left(\mathrm{NH}_{3}\right)_{2}\right]^{+}$ions, this phenomenon could be attributed to the more SERS "hot spots" in the AgNWs@PDA@AgNPs nanocobs and eventually caused a stronger electromagnetic enhancement.

The high sensitivity of SERS substrate has played an important role to measure trace components and even detect single molecules. To verify the sensitivity of the AgNWs@PDA@AgNPs nanocobs as a SERS substrate, the Raman enhancement effect was evaluated by using R6G with a concentration that ranged from $1 \times 10^{-7}$ to $1 \times 10^{-12} \mathrm{M}$. As it was depicted in Fig. 7a, with the concentration of R6G from $1 \times$ $10^{-7}$ to $1 \times 10^{-12} \mathrm{M}$, the peak intensity gradually decreased. The correlation between R6G concentration and Raman peak intensity was a good linear relationship (Fig. S4a†), and identical to the results by Qu et al. ${ }^{39}$ When the concentration of R6G molecules was lower to $1 \times 10^{-12} \mathrm{M}$, the characteristic peaks at $612,1185,1360$ and $1650 \mathrm{~cm}^{-1}$ could be still clearly observed (Fig. 7b), which revealed that the SERS substrate based on AgNWs@PDA@AgNPs nanocobs possessed a high sensitivity. For comparison, as the PDA layer reduced to about $10 \mathrm{~nm}$ thickness, the R6G detection limit on AgNWs@PDA and AgNWs@PDA@AgNPs substrates were also investigated. From Fig. S5, $\uparrow$ although the limit detection of AgNWs@PDA was down to $10^{-9} \mathrm{M}$, lower than the thicker ones (Fig. S5a $\uparrow$ ). However, the AgNWs@PDA@AgNPs based on AgNWs@PDA with PDA layer $10 \mathrm{~nm}$ could detect the concentration of R6G was down to 

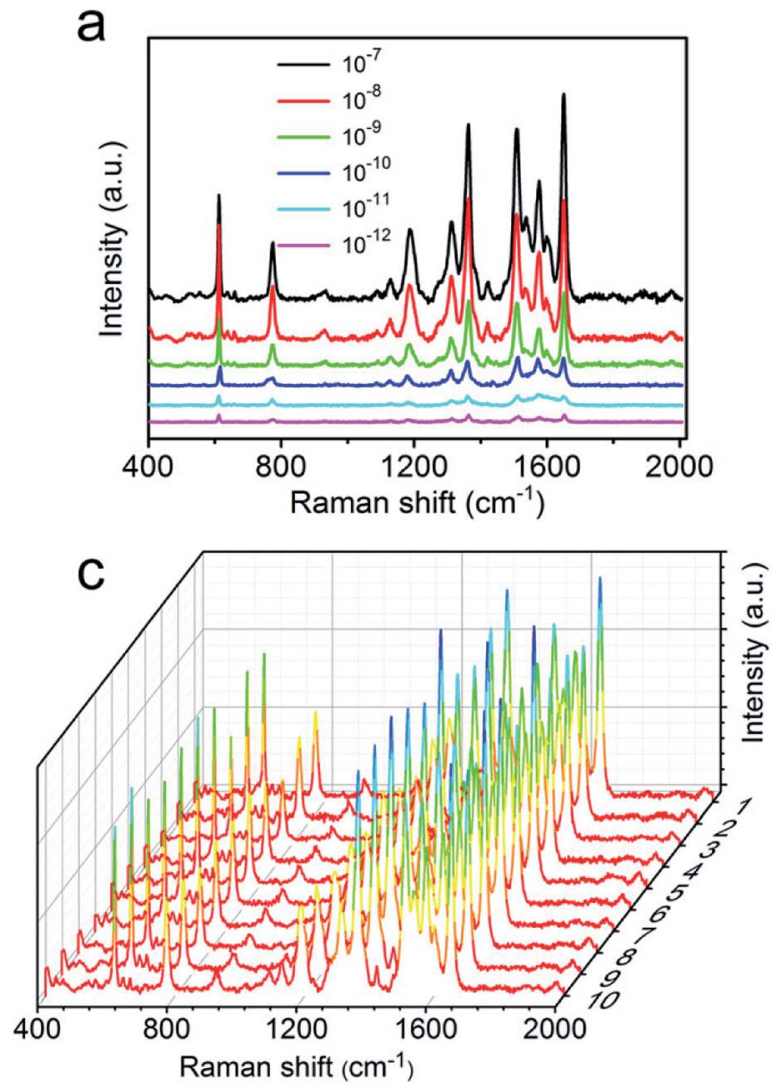

b

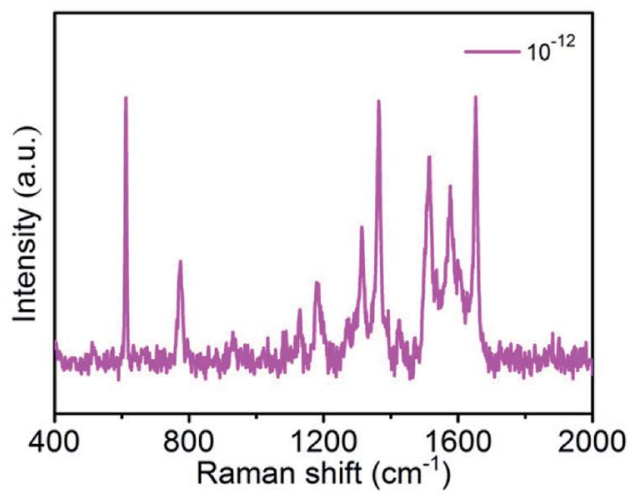

d

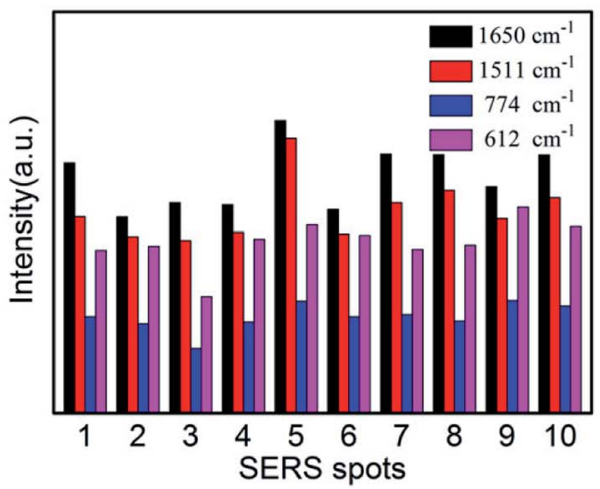

Fig. 7 (a) Raman spectra of R6G with the concentration from $1 \times 10^{-7}$ to $1 \times 10^{-12} \mathrm{M}$ on AgNWs@PDA@AgNPs, (b) the magnified SERS spectrum of R6G molecules with concentration at $1 \times 10^{-12} \mathrm{M}$, (c) SERS spectra of R6G at a concentration of $1 \times 10^{-6} \mathrm{M}$ collected from 10 random points, (d) SERS intensity changed at 612, 774, 1511 and $1650 \mathrm{~cm}^{-1}$

$10^{-11} \mathrm{M}$, higher than the thicker ones (Fig. S5b $\dagger$ ), which was due to few AgNPs on the AgNWs@PDA@AgNPs nanocobs.

Moreover, the high sensitivity of this SERS substrate was further evaluated by means of the enhancement factor, and it could be calculated from the peak intensity ratio between the normal Raman spectrum and SERS spectrum of R6G. The detail calculation procedure of enhancement factor was further described in the ESI and Fig. S4b. $\dagger$ According to the vibration absorption band at $612 \mathrm{~cm}^{-1}$, the enhancement factor was achieved by $6.49 \times 10^{7}$, and these results were comparable to the SERS substrate fabricated by an advanced nanoengineering procedure. ${ }^{\mathbf{4 0 - 4 2}}$ All these results indicated that the AgNWs@PDA@AgNPs nanocobs could serve as an excellent SERS platform for detection of trace-constituent and single molecule.

As a basic analytical implement, the reproducibility and homogeneity of SERS signals were also an important criteria to evaluate the practicability of SERS substrate besides high sensitivity. To verify the SERS reproducibility, Raman signal of R6G were collected in 10 randomly points from AgNW@PDA@AgNPs substrate. From Fig. 7c, the intensity of typical absorption bands, such as at $612,774,1511$ and $1650 \mathrm{~cm}^{-1}$, exhibited high uniformity, which revealed that the AgNWs@PDA@AgNPs nanocobs could provide excellent SERS reproducibility. ${ }^{38,43}$ Moreover, the results in Fig. 7d displayed that the relative standard deviation (RSD) values of Raman vibration at $612,774,1511$ and $1650 \mathrm{~cm}^{-1}$ were about $14.89 \%$, $16.46 \%, 15.28 \%$ and $14.55 \%(\leq 20 \%)$ respectively. In this study, a point-by-point Raman spectra of R6G were collected on the random $20 \times 20 \mu^{2}$ area at $2 \mu \mathrm{m}$ intervals on AgNWs@PDA@AgNPs substrate. Fig. S6† showed the basic vibrations of R6G molecules, and the SERS spectra results confirmed that the distribution of the Raman enhancement effect in the whole test area was uniform, and the RSD of all pixels less than $20 \%$, satisfying the requirements of uniform reinforced substrate. ${ }^{44-46}$ These values indicated that the SERS substrate based on AgNWs@PDA@AgNPs nanocobs designed by our approach was able to provide uniform SERS signals and reliably reproducibility.

Furthermore, the SERS stability of as-synthesized AgNWs@PDA@AgNPs nanocobs was also inspected. Compared the typical SERS absorption peaks of the fresh samples with those even stored for more than five months in Fig. 8a and b, no significant change of the SERS signal was observed, revealing the high stability of the SERS substrates based on AgNWs@PDA@AgNPs nanocobs fabricated by our strategy. As a result, the responding AgNWs@PDA@AgNPs nanocobs could achieve a high sensitivity, super reproducibility and excellent stability simultaneously. 

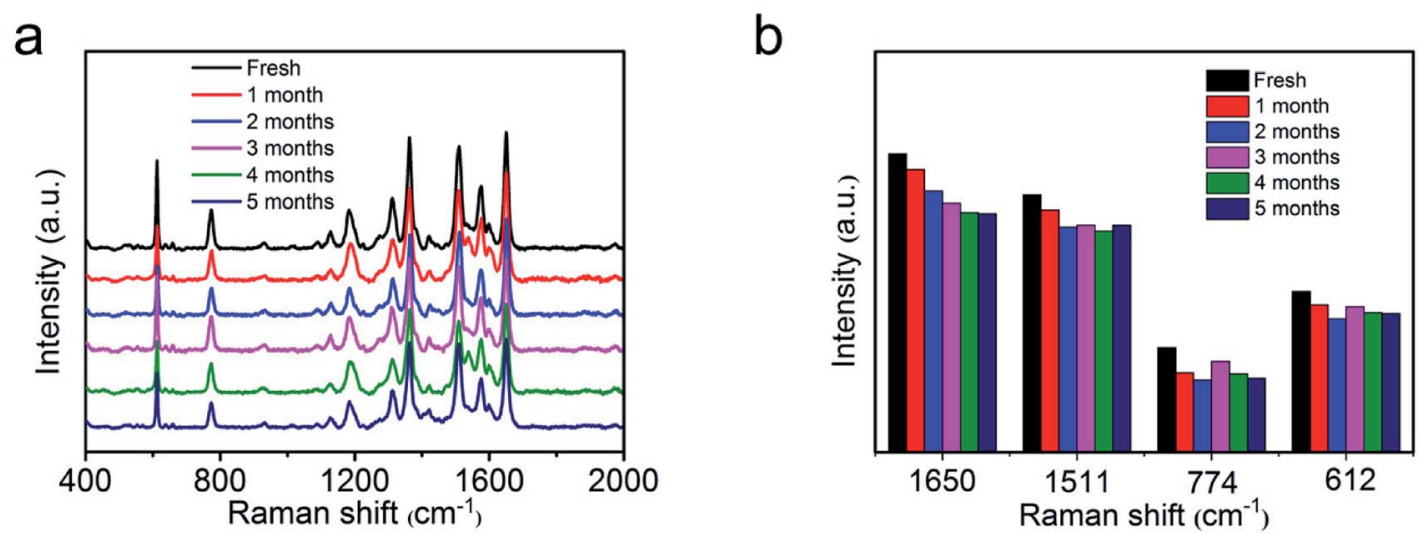

Fig. 8 (a) Raman spectra of R6G molecules on the fresh AgNWs@PDA@AgNPs nanocobs and those during stored for five months, (b) the intensity variation of typical R6G Raman peaks in the histogram.

\section{Conclusions}

A facile method was proposed to controllably fabricate a series of novel AgNWs@PDA@AgNPs core-shell nanocobs based on a mussel-inspired PDA molecule and achieved a higher sensitive detection of SERS. Through an in situ reduction role of PDA molecules, abundant AgNPs were reduced and grown on the AgNWs@PDA surface. By regulating the concentration of $\left[\mathrm{Ag}\left(\mathrm{NH}_{3}\right)_{2}\right]^{+}$ions and the PDA molecular layer on the AgNWs@PDA surface, the morphology of AgNWs@PDA@AgNPs core-shell nanocobs could be fairly controlled. Benefited from the synergistic role of AgNPs, the AgNWs@PDA@AgNPs coreshell nanocobs exhibited high SERS sensitivity for the detection of trace R6G and limit of detection could be lower to $1 \times 10^{-12} \mathrm{M}$. Moreover, as a SERS substrate, the AgNWs@PDA@AgNPs coreshell nanocobs demonstrated excellent reproducibility and superior stability. This research would provide a new strategy to build the SERS-active substrates for highly sensitive detection, and demonstrate a great potential in manufacture of more comprehensive functional SERS sensors.

\section{Conflicts of interest}

There are no conflicts to declare.

\section{Acknowledgements}

The authors would like to thank the National Nature Science Foundation (21303091, 51572134), the Nature Science Foundation of Shandong Province (ZR2018MB017), the National College Students' innovation and entrepreneurship training program (XJ201710431037, 201810431021), and the Key Research and Development Program of Shandong Province (2018GGX102023).

\section{Notes and references}

1 Y. Chen, F. Ge, S. Guang and Z. Cai, Appl. Surf. Sci., 2018, 436, 111-116.
2 T. Liyanage, A. Rael, S. Shaffer, S. Zaidi, J. V. Goodpaster and R. Sardar, Analyst, 2018, 143, 2012-2022.

3 G. Demirel, H. Usta, M. Yilmaz, M. Celik, H. A. Alidagi and F. Buyukserin, J. Mater. Chem. C, 2018, 6, 5314-5335.

4 J. Chen, Y. Huang, P. Kannan, L. Zhang, Z. Lin, J. Zhang, T. Chen and L. Guo, Anal. Chem., 2016, 88, 2149-2155.

5 J. N. Anker, W. P. Hall, O. Lyandres, N. C. Shah, J. Zhao and R. P. Van Duyne, Nat. Mater., 2008, 7, 442.

6 M. Zhang, J. Meng, D. Wang, Q. Tang, T. Chen, S. Rong, J. Liu and Y. Wu, J. Mater. Chem. C, 2018, 6, 1933-1943.

7 C.-Y. Li, M. Meng, S.-C. Huang, L. Li, S.-R. Huang, S. Chen, L.-Y. Meng, R. Panneerselvam, S.-J. Zhang, B. Ren, Z.-L. Yang, J.-F. Li and Z.-Q. Tian, J. Am. Chem. Soc., 2015, 137, 13784-13787.

8 G. Lu, H. Yuan, L. Su, B. Kenens, Y. Fujita, M. Chamtouri, M. Pszona, E. Fron, J. Waluk, J. Hofkens and H. Uji-i, J. Phys. Chem. Lett., 2017, 8, 2774-2779.

9 S. Gwo, H.-Y. Chen, M.-H. Lin, L. Sun and X. Li, Chem. Soc. Rev., 2016, 45, 5672-5716.

10 H. Hou, P. Wang, J. Zhang, C. Li and Y. Jin, ACS Appl. Mater. Interfaces, 2015, 7, 18038-18045.

11 Z. Li, M. Wang, Y. Jiao, A. Liu, S. Wang, C. Zhang, C. Yang, Y. Xu, C. Li and B. Man, Sens. Actuators, B, 2018, 255, 374383.

12 X. D. Tian, Y. Lin, J. C. Dong, Y. J. Zhang, S. R. Wu, S. Y. Liu, Y. Zhang, J. F. Li and Z. Q. Tian, Adv. Opt. Mater., 2017, 5, 1700581.

13 S. J. Lee, A. R. Morrill and M. Moskovits, J. Am. Chem. Soc., 2006, 128, 2200-2201.

14 C. Wang, B. Liu and X. Dou, Sens. Actuators, B, 2016, 231, 357-364.

15 M. S. Goh, Y. H. Lee, S. Pedireddy, I. Y. Phang, W. W. Tjiu, J. M. R. Tan and X. Y. Ling, Langmuir, 2012, 28, 14441-14449.

16 C. Du, Y. You, T. Chen, Y. Zhu, H. Hu, D. Shi, H. Chen and Z. Shen, Plasmonics, 2011, 6, 761.

17 M. Chen, I. Y. Phang, M. R. Lee, J. K. W. Yang and X. Y. Ling, Langmuir, 2013, 29, 7061-7069. 
18 M. L. Tran, S. P. Centeno, J. A. Hutchison, H. Engelkamp, D. Liang, G. Van Tendeloo, B. F. Sels, J. Hofkens and H. Uji-i, J. Am. Chem. Soc., 2008, 130, 17240-17241.

19 S.-Y. Zhang, J.-W. Liu, C.-L. Zhang and S.-H. Yu, Nanoscale, 2013, 5, 4223-4229.

20 C. Chen, J. Hao, L. Zhu, Y. Yao, X. Meng, W. Weimer and Q. K. Wang, J. Mater. Chem. A, 2013, 1, 13496-13501.

21 C. Tian, C. Ding, S. Liu, S. Yang, X. Song, B. Ding, Z. Li and J. Fang, ACS Nano, 2011, 5, 9442-9449.

22 L.-M. Chen and Y.-N. Liu, J. Raman Spectrosc., 2012, 43, 986991.

23 S. J. Lee, J. M. Baik and M. Moskovits, Nano Lett., 2008, 8, 3244-3247.

24 A. Tao, F. Kim, C. Hess, J. Goldberger, R. He, Y. Sun, Y. Xia and P. Yang, Nano Lett., 2003, 3, 1229-1233.

25 F. Hao and P. Nordlander, Appl. Phys. Lett., 2006, 89, 103101. 26 M. W. Knight, N. K. Grady, R. Bardhan, F. Hao, P. Nordlander and N. J. Halas, Nano Lett., 2007, 7, 23462350.

27 H. Wei, F. Hao, Y. Huang, W. Wang, P. Nordlander and H. Xu, Nano Lett., 2008, 8, 2497-2502.

28 R. Gunawidjaja, S. Peleshanko, H. Ko and V. V. Tsukruk, Adv. Mater., 2008, 20, 1544-1549.

29 H. Lee, S. M. Dellatore, W. M. Miller and P. B. Messersmith, Science, 2007, 318, 426-430.

30 J. Zhou, B. Duan, Z. Fang, J. Song, C. Wang, P. B. Messersmith and H. Duan, Adv. Mater., 2014, 26, 701-705.

31 H. Yang, Y. Lan, W. Zhu, W. Li, D. Xu, J. Cui, D. Shen and G. Li, J. Mater. Chem., 2012, 22, 16994-17001.

32 Y. Liu, K. Ai and L. Lu, Chem. Rev., 2014, 114, 5057-5115.
33 K. E. Korte, S. E. Skrabalak and Y. Xia, J. Mater. Chem., 2008, 18, 437-441.

34 M. B. Gebeyehu, T. F. Chala, S.-Y. Chang, C.-M. Wu and J.-Y. Lee, RSC Adv., 2017, 7, 16139-16148.

35 X. Xiao, J. Nogan, T. Beechem, G. A. Montano, C. M. Washburn, J. Wang, S. M. Brozik, D. R. Wheeler, D. B. Burckel and R. Polsky, Chem. Commun., 2011, 47, 9858-9860.

36 L. Yang, J. Hu, L. He, J. Tang, Y. Zhou, J. Li and K. Ding, Chem. Eng. J., 2017, 327, 694-704.

37 Z. Zhang and W. Zhu, Appl. Surf. Sci., 2015, 333, 214-219.

38 R. Anbazhagan, A. Vadivelmurugan, H.-C. Tsai and R.-J. Jeng, J. Mater. Chem. C, 2018, 6, 1071-1082.

39 L.-L. Qu, D.-W. Li, J.-Q. Xue, W.-L. Zhai, J. S. Fossey and Y.-T. Long, Lab Chip, 2012, 12, 876-881.

40 H. Zhulin, M. Guowen, H. Qing, C. Bin, Z. Chuhong and Z. Zhuo, J. Raman Spectrosc., 2013, 44, 240-246.

41 H. Zhulin, M. Guowen, H. Qing, Y. Yajun, Z. Chuhong and T. Chaolong, Adv. Mater., 2010, 22, 4136-4139.

42 Z. Chuhong, M. Guowen, Z. Peng, H. Qing, L. Zhongbo, H. Xiaoye, W. Xiujuan, H. Zhulin, L. Fadi and W. Nianqiang, Adv. Mater., 2016, 28, 4871-4876.

43 Q. Ding, Y. Ma, Y. Ye, L. Yang and J. Liu, J. Raman Spectrosc., 2013, 44, 987-993.

44 J. Pilipavicius, R. Kaleinikaite, M. Pucetaite, M. Velicka, A. Kareiva and A. Beganskiene, Appl. Surf. Sci., 2016, 377, 134-140.

45 Y. Xue, F. Scaglione, P. Rizzi and L. Battezzati, Appl. Surf. Sci., 2017, 426, 1113-1120.

46 R. Dasari and F. P. Zamborini, Anal. Chem., 2016, 88, 675681. 